\title{
TERNARY DERIVATIONS OF NEST ALGEBRAS
}

\author{
AJdA FoŠnER AND HOGER GHAHRAMANI
}

\begin{abstract}
Suppose that $\mathscr{X}$ is a (real or complex) Banach space, $\operatorname{dim} \mathscr{X} \geqslant 2$, and $\mathscr{N}$ is a nest on $\mathscr{X}$, with each $N \in \mathscr{N}$ is complemented in $\mathscr{X}$ whenever $N_{-}=N$. A ternary derivation of $A \lg \mathscr{N}$ is a triple of linear maps $(\gamma, \delta, \tau)$ of $A \lg \mathscr{N}$ such that $\gamma(A B)=\delta(A) B+A \tau(B)$ for all $A, B \in A \lg \mathscr{N}$. We show that for linear maps $\delta, \tau$ on $A \lg \mathscr{N}$ there exists a unique linear map $\gamma: A \lg \mathscr{N} \rightarrow A \lg \mathscr{N}$ defined by $\gamma(A)=R A+A T$ for some $R, T \in A \lg \mathscr{N}$ such that $(\gamma, \delta, \tau)$ is a ternary derivation of $A \lg \mathscr{N}$ if and only if $\delta, \tau$ satisfy $\delta(A) B+A \tau(B)=0$ for any $A, B \in$ $A \lg \mathscr{N}$ with $A B=0$. We also prove that every ternary derivation on $A \lg \mathscr{N}$ is an inner ternary derivation. Our results are applied to characterize the (right or left) centralizers and derivations through zero products, local right (left) centralizers, right (left) ideal preserving maps and local derivations on nest algebras.
\end{abstract}

Mathematics subject classification (2010): 47L35, 47B47, 16W25.

Keywords and phrases: Nest algebra, ternary (inner) derivation.

\section{REFERENCES}

[1] M. Ashraf, Sh. Ali And C. Haetinger, On derivations in rings and their applications, Aligarh Bull. Maths, 25 (2006), 79-107.

[2] A. BARARi, B. FAdAeE And H. Ghahramani, Linear Maps on Standard Operator Algebras Characterized by Action on Zero Products, Bull. Iran. Math. Soc. 45 (2019), 1573-1583.

[3] D. M. Barquero, C. M. GonZÁlez, J. SÁncheZ-Ortega And M. VAndeyar, Ternary mappings of triangular algebras, arXiv:1905.13265 (2019).

[4] D. BENKOVIČ AND M. GRAŠIČ, Generalized derivations on unital algebras determined by action on zero products, Linear Algebra Appl. 445 (2014), 347-368.

[5] M. BREŠAR, Characterizing homomorphisms, derivations and multipliers in rings with idempotents, Proc. Roy. Soc. Edinb. Sect. A. 137 (2007), 9-21.

[6] H. ChenguUn And H. Deguang, Derivations and isomorphisms of certain reflexive operator algebras, Acta Math. Sinica (in Chinese), 14 (1998), 105-112.

[7] H. Deguang, The first cohomology groups of nest algebras of normed spaces, Proc. Amer. Math. Soc. 118 (1993), 1147-1149.

[8] H. Deguang And W. Shuyun, Local derivations of nest algebras, Proc. Amer. Math. Soc. 123 (1995), 3095-3100.

[9] B. FADAEe AND H. Ghahramani, Jordan left derivations at the idempotent elements on reflexive algebras, Publ. Math. Debrecen, 92 (2018), 261-275.

[10] H. Ghahramani, On rings determined by zero products, J. Algebra and appl. 12 (2013), 1-15.

[11] H. Ghahramani, On derivations and Jordan derivations through zero products, Operator and Matrices, 4 (2014), 759-771.

[12] H. GHAHRAMANI, Linear maps on group algebras determined by the action of the derivations or anti-derivations on a set of orthogonal elements, Results in Mathematics, 73 (2018), 132-146.

[13] H. Ghahramani, Characterizing Jordan maps on triangular rings through commutative zero products, Mediterr. J. Math. (2018) 15: 38, https://doi .org/10.1007/s00009-018-1082-3.

[14] H. Ghahramani AND Z. PAn, Linear maps on $\star$-algebras acting on orthogonal elements like derivations or anti-derivations, Filomat, 13 (2018), 4543-4554.

[15] H. GHAhramani AND S. SATTARI, Characterization of reflexive closure of some operator algebras acting on Hilbert $C^{*}$-modules, Acta Math. Hungar. 157 (1) (2019), 158-172. 
[16] H. Ghahramani, Left ideal preserving maps on triangular algebras, Iran. J. Sci. Technol. Trans. Sci. (2019), https://doi .org/10.1007/s40995-019-00794-2.

[17] The HADWIN Lunch Bunch, Local multiplications on algebras spanned by idempotents, Linear Multilinear Algebra, 37 (1994), 259-263.

[18] D. Hadwin And J. KerR, Local multiplications on algebras, J. Pure Appl. Algebra, 115 (1997), 231-239.

[19] D. Hadwin And J. Li, Local derivations and local automorphisms, J. Math. Anal. Appl. 290 (2004), $702-714$.

[20] D. HADWIN AND J. LI, Local derivations and local automorphisms on some algebras, J. Operator Theory, 60 (2008), 29-44.

[21] J. C. Hou AND X. L. Zhang, Ring isomorphisms and linear or additive maps preserving zero products on nest algebras, Linear Algebra Appl. 387 (2004), 343-360.

[22] C. JimenÉz-Gestal AND J. M. PÉREZ-IZQuierdo, Ternary derivations of generalized CaylelDickson algebras, Comm. Algebra, 31 (10) (2003), 5071-5094.

[23] C. JimenÉZ-Gestal ANd J. M. PÉREZ-IZQuierdo, Ternary derivations of finite-dimensional real division algebras, Linear Algebra Appl. 428 (2008), 2192-2219.

[24] B. E. Johnson, Centralisers and operators reduced by maximal ideals, J. London Math. Soc. 43 (1968), 231-233.

[25] E. KATSOULIS, Local maps and the representation theory of operator algebras, Trans. Amer. Math. Soc. 368 (2016), 5377-5397.

[26] T.-K. LEE, Generalized skew derivations characterized by acting on zero products, Pacific J. Math. 216 (2004), 293-301.

[27] L. LIU, Characterization of centralizers on nest subalgebras of von Neumann algebras by local action, Linear and Multilinear Algebra, 64 (2016), 383-392.

[28] L. LiU, On local Lie derivations of generalizedmatrix algebras, Banach J. Math. Analysis, (2019), https://doi.org/10.1007/s43037-019-00018-0.

[29] J. Li AND Z. PAN, Annihilator-preserving maps, multipliers, and derivations, Linear Algebra Appl. 432 (2010), 5-13.

[30] J. M. PÉREZ-IzQuierdo, Unital algebras, Ternary derivations, and local triality, Algebras, representations and applications, Contemp. Math. Amer. Math. Soc. Providence, RI. 483 (2009), 205-220 RI.

[31] X. F. QI AND J. C. Hou, Characterizing centralizers and generalized derivations on triangular algebras by acting on zero product, Acta. Math. Sin.-English Ser. 29 (2013), 1245-1256.

[32] A. I. SHESTAKov, Ternary derivations of separable associative and Jordan algebras, Siberian Math. J. 53(5) (2012), 943-956.

[33] A. I. SheStAKov, Ternary derivations of Jordan superalgebras, Algebra Logika, 53 (4) (2014), 505 540 (in Russian), Algebra Logic, 53 (4) (2014), 323-348 (in English).

[34] N. K. Spanoudakis, Generalization of certain nest algebras results, Proc. Amer. Math. Soc. 115 (1992), 711-723.

[35] W. S. XU, R. L. AN AND J. C. Hou, Equivalent characterization of centralizers on $B(\mathscr{H})$, Acta Math. Sin. English Ser. 32 (2016), 1113-1120.

[36] J.-H. Zhang, G.-X. Ji AND H.-X. CAO, Local derivations of nest subalgebras of von Neumann algebras, Linear Algebra Appl. 392 (2004), 61-69.

[37] V. N. ZHELYABIN AND A. I. SHESTAKOV, Alternative and Jordan algebras admitting ternary derivations with invertible values, Sib. Élektron. Mat. Izv. 14 (2017), 1505-1523. 